DISEÑO DE LA INDUCCIÓN GENERAL AJUSTADA AL MODELO DE COMPETENCIAS DE UNA EMPRESA DEL SECTOR BANCARIO

Presentado por: Cristian Alejandro Pineda Ávila

Tutor: Myriam Sosa Espinosa

UNIVERSIDAD SANTO TOMÁS

FACULTAD DE ADMINISTRACIÓN DE EMPRESAS

BOGOTÁ-COLOMBIA

2018 


\section{DISEÑO DE LA INDUCCIÓN GENERAL AJUSTADA AL MODELO DE COMPETENCIAS DE UNA EMPRESA DEL SECTOR BANCARIO}

Trabajo de grado presentado para obtener el título de profesional en administración de empresas

Presentado por:

Cristian Alejandro Pineda Ávila

Tutor:

Myriam Sosa Espinosa

BOGOTÁ, 2018 


\section{Tabla de contenido}

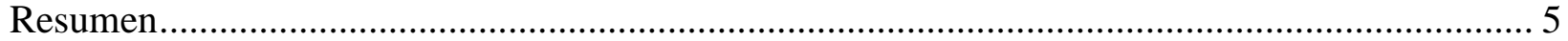

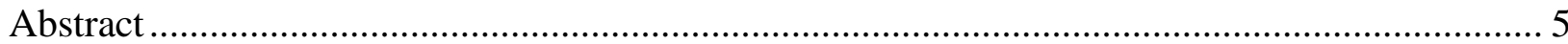

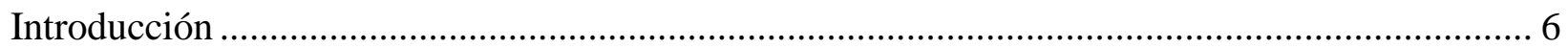

Planteamiento del Problema ............................................................................................... 7

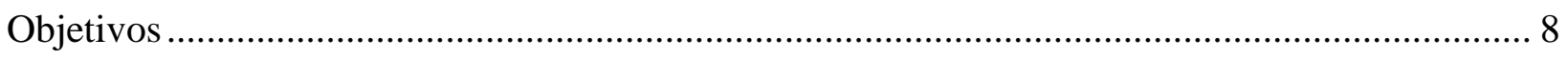

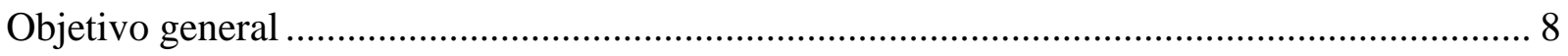

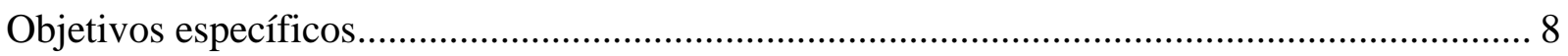

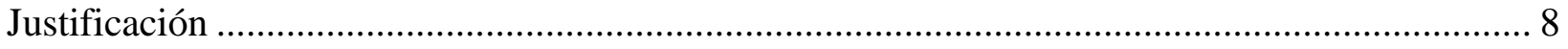

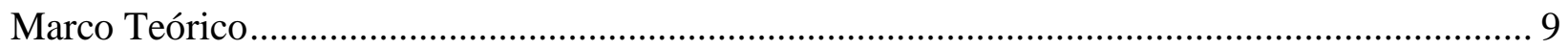

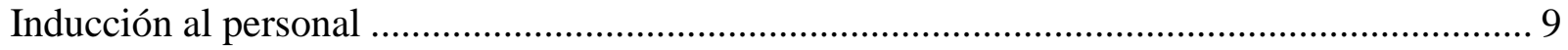

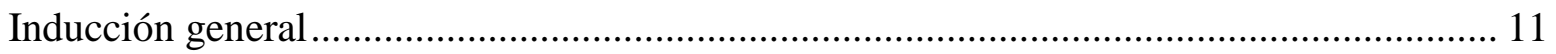

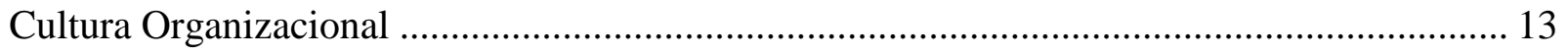

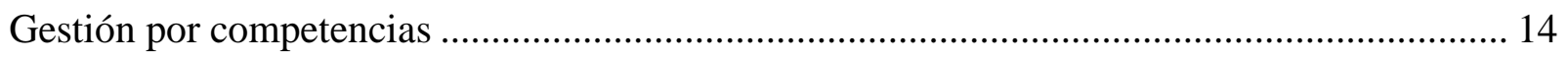

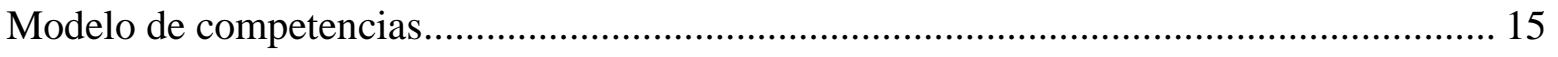

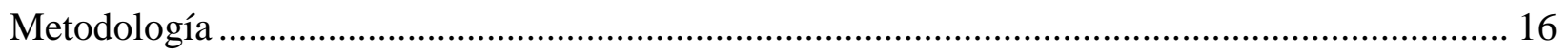

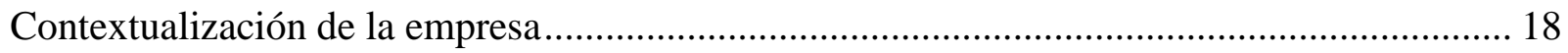

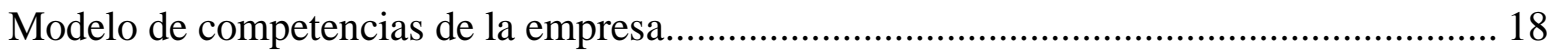

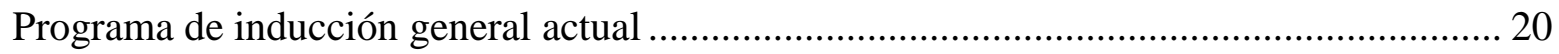

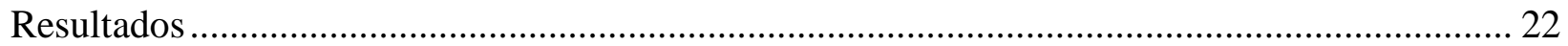

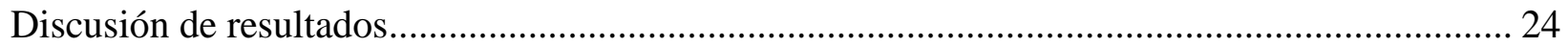

Propuesta para el nuevo programa de inducción general ................................................. 25

Contenido de la inducción general ............................................................................. 25

Información entregada en el manual de inducción virtual ............................................. 30 


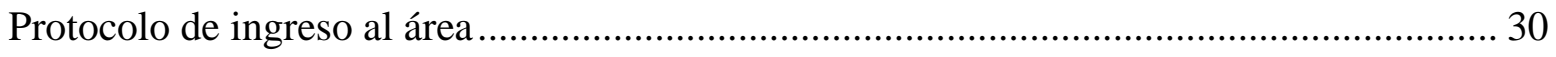

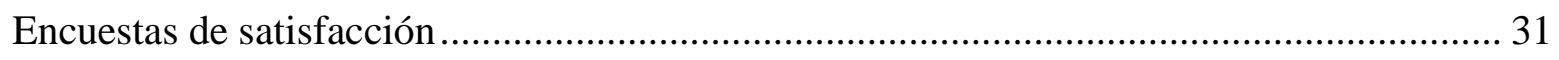

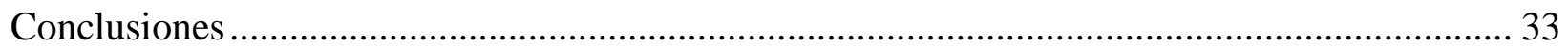

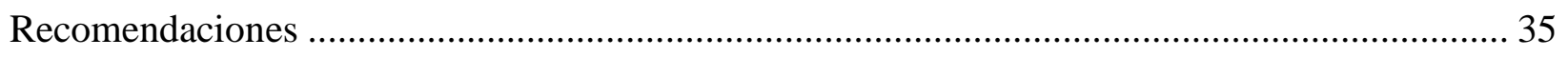

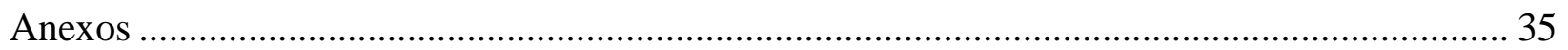

Anexo 1: Herramientas de recolección de información (Entrevista) .......................................... 35

Anexo 2: Herramientas de recolección de información (Encuesta) ............................................. 36

Anexo 3: Evidencias de las encuestas aplicadas …………………………………….......... 37

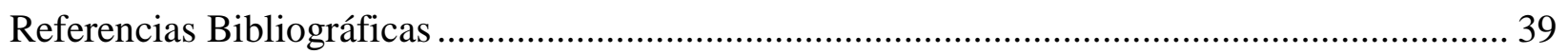

\section{Lista de tablas}

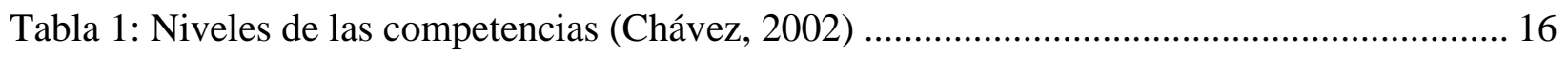

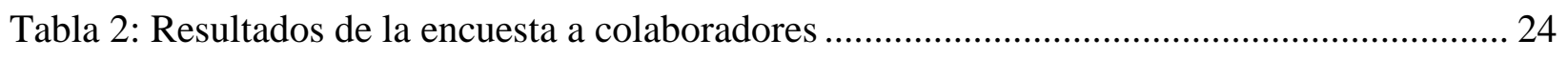

Tabla 3: Propuesta, metodología de la inducción general ................................................................ 25

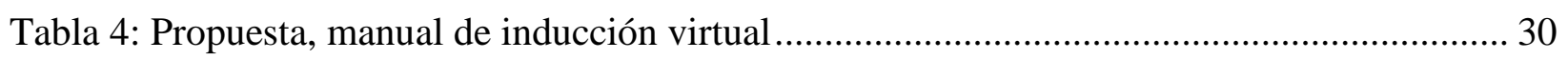

Tabla 5: Encuesta de satisfacción a la inducción general .............................................................. 31

Tabla 6: Encuesta de satisfacción a la inducción específica........................................................... 32

\section{Lista de figuras}

Figura 1: Subsistemas de Talento Humano. (Alles, 2016) ……………………………............ 12

Figura 2: Actividades del assesment center para la propuesta....................................................... 28

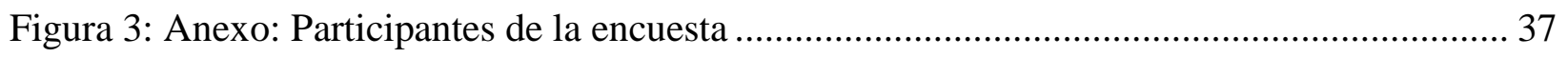

Figura 4: Pregunta 2 "Comprendí la cultura organizacional de la empresa con el proceso de

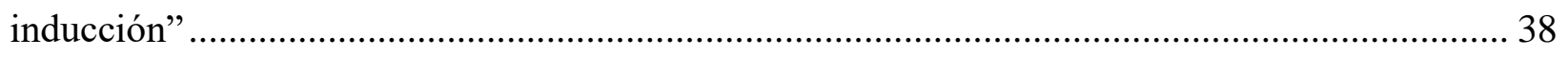

Figura 5: Pregunta 4: "Se cumplieron mis expectativas en la empresa generadas en la inducción 


\title{
Resumen
}

En el presente trabajo se describe una propuesta de un programa de inducción general para una empresa del sector bancario ubicada en la ciudad de Bogotá. La inducción general es el primer paso de los colaboradores dentro de la organización y la empresa en estudio quiere que ese día se les genere un alto impacto, logrando desde el principio compromiso y sentido de pertenencia. Para diseñar una inducción general que cumpla con este objetivo se recolectó la información necesaria realizando entrevistas a los miembros del área de formación y aplicando encuestas a los trabajadores que recibieron la inducción en fechas recientes. Como resultado se presenta una estructura de la inducción general que dinamiza y simplifica el proceso haciendo énfasis en los aspectos de la empresa que representan beneficios e interioriza las competencias que se esperan del nuevo personal.

Palabras claves: Inducción, modelo de competencias, motivación, cultura organizacional.

\begin{abstract}
In this document, is described a proposal to a general induction program from a company in the banking sector located in Bogota. The general induction is the first step of the workers in the organization; nowadays the company wants to generate a good impact in its workers this day, achieving from the beginning commitment and sense of belonging. Ins this regard for design a general induction to reach this objective information was gathered conducting interviews to the work area of training's members and polls to the workers who recently have been in the general induction. Finally is presented a structure of the general induction more dynamic and simplified, emphasizing in the positive aspects and working with the skills required in the new workers.
\end{abstract}

Key words: Induction, model skills, motivation, organizational culture. 


\section{Introducción}

La inducción al nuevo personal en una organización corresponde al primer esfuerzo que ésta hace para que un colaborador conozca sobre su puesto, equipo y funciones de trabajo, así como cultura y normas de la empresa, amoldando sus comportamientos a lo que ésta necesita; los nuevos colaboradores, usualmente, suelen decidir durante los primeros seis meses de trabajo si quieren continuar allí por un tiempo duradero o no; por lo que las organizaciones se esfuerzan en que esta primera impresión genere en sus miembros sentido de pertenencia. (Mondy, 2010).

La presente investigación se realizó en una empresa del sector bancario ubicada en la ciudad de Bogotá, a la cual no nos referimos explícitamente por su nombre por privacidad, que plantea realizar cambios en su programa de inducción general a los nuevos colaboradores que les permita conocer los parámetros de su empleo y les genere una buena primera impresión formando sentido de identidad, ya que; una correcta inducción general es una práctica de Talento Humano que si se hace de forma adecuada logra generar sentido de pertenencia en los trabajadores (Alles, 2008). La inducción general se debe ajustar al modelo de gestión por competencias, para que de esta forma se dé inicio a la apropiación de competencias en alineación con los objetivos organizacionales. (Alles, 2016).

Se dió la oportunidad de presentar una propuesta para el programa que responda a la situación planteada, para lo cual se realizó una investigación cualitativa de tipo descriptivo que incluye el análisis de la inducción general actual y la recolección de la información necesaria de las personas involucradas con el proceso (los miembros del área de formación y los colaboradores que han recibido recientemente inducción general); con el objetivo de diseñar un programa de inducción al personal, ajustado al modelo de gestión por competencias para incrementar el impacto del proceso en los nuevos colaboradores.

Debe generar al nuevo colaborador una expectativa positiva de la organización asegurando que el tiempo que estará trabajando allí será enriquecedor, desde la empresa y el área a la que se dirige; por lo tanto, la inducción es un proceso vinculado entre el área de Talento Humano y su jefe inmediato junto con su equipo de trabajo, siendo con estas últimas personas con quien pasará la mayor parte del tiempo de su estadía en la empresa. (Mondy, 2010: p. 211). 


\section{Planteamiento del Problema}

La inducción a los nuevos colaboradores se define como: “(...) parte del proceso de socialización del empleador para los empleados nuevos. La inducción es el proceso permanente para imbuir en todos los empleados las actitudes, normas, valores y patrones de conducta que prevalecen y son esperados por la organización y sus departamentos” (Dessler, 2001: p. 249). Las organizaciones seleccionan a los administradores adecuados, sin embargo, muchas veces ignoran que tienen necesidades al momento de ingresar a trabajar, por lo que los primeros días suelen ser decisivos en la adecuada integración de las personas a su cargo para la continuidad del funcionamiento de la empresa. (Weihrich y Koontz, 1998: p. 409)

Nos encontramos frente a una empresa del sector bancario ubicada en la ciudad de Bogotá, a la cual no nos referimos explícitamente por su nombre por políticas de privacidad y confidencialidad. La empresa cuenta con una larga trayectoria y con estructura de Talento Humano definida. En una reunión con la directora del área de formación, se encontró que la empresa cuenta con un proceso de inducción general que ha dado los resultados apropiados y cumple con los requerimientos de la compañía, que son, en principio que los colaboradores conozcan los parámetros de su empleo y trabajen en función del modelo de gestión por competencias. Sin embargo, en un proceso de mejora continua, se presentan propuestas para realizar cambios en ella, deseando optimizar el impacto que genera en las personas que ingresan a la organización.

El presente trabajo se orientó a diseñar un nuevo programa que da respuesta a los requerimientos de la organización, ajustándose al modelo de gestión por competencias y mejorando la primera impresión que genera la empresa en los nuevos colaboradores, respondiendo a la pregunta:

¿Qué programa de inducción general del personal se ajusta al modelo de gestión por competencias en una empresa del sector bancario ubicada en la ciudad de Bogotá? 


\section{Objetivos}

\section{Objetivo general}

Diseñar un programa de inducción general al personal en una empresa del sector bancario ubicada en la ciudad de Bogotá, ajustado a su modelo de gestión por competencias para incrementar el impacto del proceso en los nuevos colaboradores.

\section{Objetivos específicos}

I. Hacer un diagnóstico del programa de inducción general en una empresa del sector bancario en Bogotá para identificar sus oportunidades de mejora.

II. Analizar el modelo de competencias de una empresa del sector bancario en Bogotá para integrarlo en la propuesta del programa de inducción general.

III. Estructurar el nuevo programa de inducción general en una empresa del sector bancario en Bogotá para mejorar el impacto en los colaboradores.

\section{Justificación}

Un buen plan de inducción genera bastantes beneficios para una organización, entre ellos: el crear en los colaboradores verdadero sentido de pertenencia e identidad, hecho que lleva al compromiso, a establecer objetivos conjuntos entre empresa y empleado y una actitud favorable hacia la compañía, además de generar en el trabajador identidad con el grupo y habilidades de comunicación rápidamente. (Grados, 2012).

En el área de formación de una entidad del sector bancario ubicada en la ciudad de Bogotá, de la cual no tenemos autorización para referirnos explícitamente por su nombre por privacidad, se planteó la necesidad de realizar cambios en su programa de inducción general ya que es un 
proceso bastante extenso y expositivo, además, que no se cuenta con un plan de bienvenida o acoplamiento en cada área. Así que se busca que con este proceso las nuevas personas se sientan identificadas con la organización desde el primer día. El proceso de inducción brinda a los nuevos colaboradores las herramientas necesarias para dar inicio a sus labores y, dado que estas primeras impresiones suelen ser las más duraderas (Grados, 2012) se hace necesario generar un impacto positivo que garantice el mejor resultado de los colaboradores desde el inicio. El proceso se debe ajustar al modelo de gestión por competencias de la empresa para que el personal pueda apropiar la cultura organizacional desde el comienzo. Este proceso adecuadamente realizado influye positivamente en la permanencia de una persona dentro de una organización y su motivación (Mondy, 2010), con lo cual el desempeño de las funciones de un área y su impacto en el objeto social de la empresa son óptimos.

Dicho esto, se hizo conveniente presentar al área de formación de la entidad analizada una propuesta para el nuevo programa de inducción general y un protocolo de bienvenida al área que da respuesta a las necesidades que se identifican en el análisis del actual programa y a los objetivos planteados por la organización ajustándose al modelo de competencias.

\section{Marco Teórico}

\section{Inducción al personal}

Alguna de las definiciones que se le atribuye a la inducción personal nos dice que "(...) implica dotar a los nuevos empleados de información preliminar sobre la empresa, sus funciones, sus tareas y su personal", las empresas suelen contar con una estructura del programa de inducción para nuevos colaboradores que implica el darles a conocer aspectos generales como historia de la empresa, sus productos, servicios, políticas, organización, ubicación, adaptación y conductas de los trabajadores; además, de los conocimientos, capacidades y normas necesarias para su cargo. (Weihrich y Koontz, 1998: p. 409)

Con este proceso la organización busca alcanzar los siguientes objetivos: 
- Reducir la ansiedad e incertidumbre en los nuevos colaboradores.

- Reducir la rotación de personal, que se causa en un inicio porque los trabajadores se sienten ineficientes o no necesitados en la empresa.

- Ahorrar tiempo ya que se le da a conocer a los nuevos colaboradores aspectos que necesita saber antes de que llegue a su puesto de trabajo a averiguarlos.

- Dar a conocer a los nuevos colaboradores qué se espera de ellos dentro de la compañía y las expectativas que ellos pueden tener allí. (Chiavenato, 2009)

Las empresas eligen si sus procesos de inducción son extensos o bastantes concisos dependiendo de la organización; de cualquier forma, lo indispensable es entregar al colaborador la información necesaria para que pueda desempeñar de forma adecuada sus funciones, lo que incluye manuales, herramientas, procedimientos, beneficios, entre otros, además de indicar las normas, actitudes, valores y patrones de conducta que asumen los colaboradores de la organización. (Dessler, 2001: p. 249)

Una vez han culminado el proceso de selección, las personas que ingresan a la organización son los nuevos colaboradores, a los cuales se hace necesario arraigarlos a la filosofía, lenguaje habitual, estructura organizacional, costumbres, de su nuevo sitio de trabajo, deshaciéndose de hábitos y conductas que resultan indeseables para la organización y no se ajustan con su cultura organizacional, por medio de un programa que tarda de 1 a 5 días; de forma que se hace un proceso recíproco donde el nuevo colaborador y la empresa se adecúan el uno al otro de acuerdo con sus necesidades. (Chiavenato, 2009). Una buena inducción genera en los nuevos colaboradores motivación y sentido de pertenencia por la organización lo cual, a su vez, crea personas con compromiso, involucramiento e impulso por realizar sus labores de la mejor manera posible. (Alles, 2008, p. 303).

Cada compañía decide si realiza inducción a su personal o no, eso depende del tipo de negocio que maneje y la disposición que tenga para realizar inversiones en Talento Humano, por ejemplo, una organización cuyo modelo de negocio tiene miras a obtener ganancias a mediano y largo plazo, tiene en su planeación que el personal esté bastante tiempo allí e invierte en su gestión. Si se realiza un proceso adecuado de inducción se evidenciarán efectos positivos para la empresa y el colaborador, tales como generar motivación, actitud positiva y una rápida adaptación al grupo y las funciones de su cargo. (Grados, 2012). 
La inducción al nuevo personal es una responsabilidad conjunta entre el área de Talento Humano y el área al que va dirigido cada colaborador, ya que la primera se encarga de entregar a los trabajadores información de interés general, y que corresponde a todas las personas, los programas más complejos de inducción general incluyen películas y vídeos de los altos directivos dando la bienvenida. La segunda parte, en donde se le brinda al colaborador información específica sobre su puesto, sus funciones y su equipo de trabajo, de lo cual está a cargo el futuro jefe inmediato, durante este segundo proceso el jefe aprovecha para vincular al nuevo integrante con el equipo, sus nuevos compañeros y objetivos a alcanzar. (Werther y Davis, 2014)

\section{Inducción general}

Simplificando el contenido que debe contener una inducción general para nuevo personal damos por hecho que los aspectos mínimos que se deben dar a conocer son:

- Aspectos sobre la empresa:

o Misión y visión

o Organigrama

o Operaciones: Productos, volúmenes, cifras

- Aspectos geográficos

- Aspectos mundiales si es una corporación

- Políticas, normas internas, sistemas

- Comunicaciones, costumbres de la compañía: horarios, feriados especiales, etc. (Alles, 2006: p. 208)

Otro punto de vista destaca que los temas que se deben dar a conocer en el manual para el nuevo colaborador, ya que resulta importante que conozca, son los siguientes:

- Un mensaje de bienvenida

- Historia de la organización

- El modelo negocio de la organización

- El futuro del colaborador en la organización 
- Aspectos relacionados con el trabajo (Horario, descansos, faltas, fechas de pago, supervisión, lugares de trabajo, uso de herramientas como el teléfono)

- Prestaciones (Vacaciones, feriados, seguros, transporte, servicio social, etc,)

- Prestaciones especiales (Sindicatos, restaurante, gimnasio y recreación, entre otros) (Chiavenato, 2009: p, 191).

La inducción general o incorporación del personal es uno de los subsistemas del área de Talento Humano, el segundo en el orden de la cadena de valor (Tabla 1), junto con el proceso de reclutamiento y selección; siendo los encargados de cubrir adecuadamente una posición con su respectivo perfil (Alles, 2016).

Figura 1: Subsistemas de Talento Humano. (Alles, 2016)

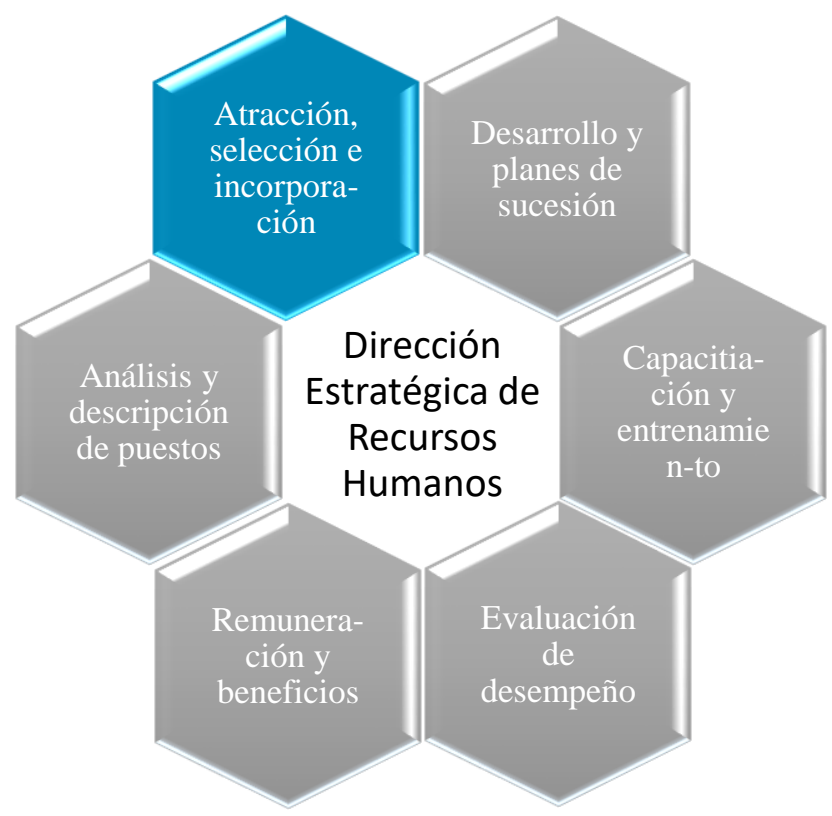

Más allá de dar información del banco como productos, servicios, organización, prestaciones, entre otros (ya que este trabajo puede hacerlo un folleto) se hace importante que en el proceso de inducción de los colaboradores se abra el espacio para solucionar dudas e incluir la socialización organizacional que implica la instrucción de las normas, conductas, nuevos relacionamientos, valores y demás componentes de la cultura organizacional; ya que todos estos aspectos suelen 
crear incertidumbre en los nuevos colaboradores. Como ejemplo de comportamientos la persona que imparte el proceso de inducción debe ser de la más alta calidad, que sirva de ejemplo ya que esta primera experiencia es importante para la futura conducta de los nuevos empleados. (Weihrich y Koontz, 1998: p,409)

Dentro del proceso de inducción también se pueden generar algunos inconvenientes, entre ellos algunos que tienen que ver con la parte de la inducción a cargo del jefe inmediato, ya que este tiende a disponer de poco tiempo o a estar tan familiarizado con los procesos que ve innecesaria una explicación, o a dejar la inducción a cargo de uno de sus compañeros. Se sugiere para un proceso de la inducción completo tener en cuenta las siguientes recomendaciones:

- Evitar dar excesiva información al nuevo colaborador, así como demasiados formatos y cuestionarios.

- No dar inicio con la parte negativa del cargo.

- No pedir inicialmente al colaborador realizar labores en las que pueda fracasar. (Werther y Davis, 2014)

\section{Cultura Organizacional}

Se puede definir como “(...) el patrón de conducta, creencias y valores que sus miembros comparten (...). Implica la adquisición y transmisión de conocimientos, creencias y patrones de conducta en el transcurso del tiempo, lo que la hace sumamente estable" (Weihrich y Koontz, 1998, p. 353). Está completamente ligado con el comportamiento, ya que genera un sistema de 'significado compartido' entre los colaboradores que los encamina por una misma ruta hacia un objetivo común por medio de características que comparten y rigen su vida laboral. (Robbins y Judge, 2006: p, 550)

La cultura organizacional debe ir de la mano con la planeación estratégica de la organización, ya que la primera es el manifiesto conductual de la segunda y su medio. De allí que los hábitos y creencias organizacionales creen motivación y compromiso en los colaboradores. Este compromiso de los colaboradores con la estrategia organizacional, además de los valores y normas son la principal base para una alta productividad, teniendo en cuenta que la gestión en las 
empresas hoy en día se enfoca hacia las personas, que son quienes desarrollan los procesos. Parte de este enfoque es lograr una buena imagen de la empresa por sus empleados con la intención de atraer y retener el mejor talento (Cuesta, 2010)

\section{Gestión por competencias}

Según D. McClelland una competencia es una característica que estando sumergida en lo profundo de un individuo se puede evidenciar en sus comportamientos observables, ya que los causa o los predice, permitiendo así, la medición de una correcta o incorrecta ejecución de una tarea o criterio específico (Ríos, Sarnataro y Soto, 2003)

La gestión por competencias es un método de gestión del Talento Humano que consiste en ajustar a la misión y la visión de la empresa con los comportamientos que se desean en los colaboradores para que se cumpla con los objetivos. La gestión por competencias correctamente definida es la alineación del Talento Humano de la empresa con su estrategia. (Alles, 2006)

Spencer y Spencer realizan una clasificación de las competencias de acuerdo con su origen, siendo así:

- De motivación: Los estímulos que una persona tiene y que la dirigen y alejan de ciertas acciones, siendo estas competencias rasgos intrínsecos que desembocen en su desempeño

- Características: Cualidades físicas y estímulos de respuesta ante determinadas situaciones

- Concepto propio: Las actitudes que derivan de la imagen que una persona tiene de sí misma.

- Conocimiento: Información que posee una persona.

- Habilidad: Destreza para desarrollar una actividad física o mental. (Alles, 2006)

El objetivo final de la gestión por competencias es lograr que todos sus colaboradores posean las capacidades necesarias para que se cumpla con la planeación (Misión, visión objetivos), por lo cual crean un modelo de competencias explicando allí el perfil ideal de cada miembro y cargo. Se sabe que cada perfil es ambicioso ya que explica cómo debe ser el colaborador "perfecto" 
para ocuparlo, sin embargo, también es alcanzable, permitiendo que se trabaje en él para lograrlo. (Alles, 2010)

Una vez una empresa asume la gestión de su personal por competencias relaciona con este método todos sus procesos, haciendo, si es necesario, las modificaciones precisas para incorporarlo en sus diseños (Alles, 2016).

\section{Modelo de competencias}

La expresión de modelo de competencias hace referencia a: "El conjunto de procesos relacionados con las personas que integran la organización y que tienen como propósito alinearlas en pos de los objetivos organizacionales" (Alles, 2016: p. 56) Allí se recogen y explican las competencias necesarias para una organización teniendo en cuenta la planeación estratégica, objetivos del cargo y cultura organizacional; presentando las competencias tanto genéricas y específicas, que necesita tener cada persona para desempeñar adecuadamente sus funciones. (Calvo, 2014).

Las organizaciones usan el modelo de competencias como base para trabajar las funciones de los subsistemas dentro del área de Talento Humano de la siguiente forma:

- Selección: Allí se garantiza que las personas que ingresan a cubrir las vacantes cumplen con las competencias necesarias para hacerlo y están en el mejor lugar posible.

- Formación: Se enseñan conocimientos desarrollan competencias conforme a las necesidades del cargo y la empresa.

- Desempeño: Se evalúa el desempeño de los colaboradores y se identifican las competencias que se pueden mejorar. (Alles, 2006)

En la elaboración e implantación de un modelo de competencias, alguna competencia puede ser asignada para varios cargos, sin embargo, dependiendo de la jerarquía o responsabilidad de cada cargo el nivel en que se debe presentar la competencia es diferente. Si tenemos dentro de un área los cargos A, B, C y D (siendo D el más pequeño y A el más alto), y a los 4 se les asigna una competencia, cabe destacar que el cargo A, debe demostrar un nivel más exigente que los 
demás, sin embargo, este cargo debe manifestar, también, los niveles que corresponden a los cargos más bajos. Se debe precisar la competencia en el nivel que se desea para asegurar el resultado y desarrollo necesario de cada persona. (Hay que tener en cuenta que el nivel 1 aplica a los cargos de menor jerarquía, mientras los de nivel 5 a los cargos de mayor jerarquía). (Chávez, 2002). Un método utilizado para ubicar cada competencia en el nivel adecuado es ubicar las funciones del cargo dentro de los siguientes criterios:

Tabla 1: Niveles de las competencias (Chávez, 2002)

\begin{tabular}{|c|c|c|}
\hline Categoría & Nivel & Función en la tarea \\
\hline \multirow{4}{*}{ Operativa } & 1 & Ejecutar \\
& 2 & Accionar \\
& Realizar \\
\cline { 2 - 3 } & \multirow{2}{*}{3} & Conducir \\
\cline { 2 - 3 } & & Mantener \\
& & Verificar \\
\hline \multirow{3}{*}{ Directiva } & 4 & Dirigir \\
& & Distribuir \\
& & Controlar \\
\cline { 2 - 3 } & & Inventar \\
& & Promover \\
& 5 & Estudiar \\
& & Establecer \\
\hline
\end{tabular}

\section{Metodología}

El presente es un estudio cualitativo, teniendo en cuenta que este se define como la recolección e interpretación de datos para dar respuesta a preguntas que se van planteando antes, durante y después de la investigación priorizando las más importantes, por lo tanto, es un proceso circular que no tiene una secuencia definida (Hernández, Fernández y Baptista, 2010: p. 7). El estudio se enfocó en reunir la información necesaria para conocer las necesidades en un nuevo programa de inducción general para una empresa del sector bancario ubicada en la ciudad de Bogotá. 
El alcance es hacia un estudio tipo descriptivo, siendo este aquel que busca la presentación de las características de un fenómeno con base a la recolección y análisis de información.

(Hernández, Fernández y Baptista 2010: p. 80). Ya que la metodología consistió en el análisis de datos recogidos de las fuentes necesarias y la descripción de un diseño de inducción general como respuesta a las necesidades encontradas.

Se realizó una inspección al modelo de gestión por competencias y al actual programa de inducción general del banco; lo cual fue consultado de documentación suministrada por la propia organización.

Para la recolección de información una de las herramientas que se usó fue une entrevista semiestructurada aplicada a la directora del área de formación y los analistas relacionados (Ver anexo 1). Este tipo de entrevista es una reunión en la cual se conversa para intercambiar información entre un entrevistador y uno o varios entrevistados, teniendo como base unas preguntas, sin embargo, el entrevistador puede agregar otras durante la entrevista si lo considera necesario. (Hernández, Fernández y Baptista 2010: p. 418). Esto con el fin de conocer el proceso de inducción general al banco, su modelo de competencias y las necesidades y expectativas del nuevo programa.

Para conocer las perspectivas de los colaboradores se diseñó y se aplicó un cuestionario tipo Likert (Ver Anexo 2); el cual consiste hacer afirmaciones sobre un tema determinado y dar el espacio para que la persona encuestada manifieste su reacción seleccionando alguna de las opciones, que pueden ser, por ejemplo: totalmente de acuerdo, de acuerdo, en desacuerdo, totalmente en desacuerdo. (Hernández, Fernández y Baptista, 2010). La población seleccionada fueron los colaboradores que habían recibido inducción general durante los seis primeros meses del año 2017 y se toma una muestra de 18 personas dentro de esta población.

Además de esto, la empresa suministró las conclusiones que obtuvo de un "Focus group" realizado en la población de colaboradores que recibieron inducción general en los años 2016 y 2017, seleccionando una muestra de 20 personas. El "focus group" o grupo de discusión es un método para la obtención de datos para una investigación cualitativa que consiste en reunir a un grupo de personas, (usualmente entre siete y doce), para consultar sus posiciones frente un tema en específico, buscando un ambiente donde puedan expresar sus opiniones cómodamente. (Juan y Roussos, 2010). 


\section{Contextualización de la empresa}

Entidad financiera con más de 30 años en el negocio que tiene como objeto social la captación y colocación de dinero en el mercado para las personas naturales, empresas y constructores; con miras a mejorar la rentabilidad, relación costo-beneficio, desarrollo del recurso humano y lealtad de los clientes por medio de estrategias que cumplen con la planeación estratégica. Tiene presencia a nivel nacional en la mayoría de las ciudades del país divididas en 4 regionales ubicadas en el norte, suroccidente, noroccidente y sur del país, en donde se incluye Bogotá, ciudad donde presenta su mayor concentración en cuanto a personal, oficinas y clientes.

\section{Misión}

Dar satisfacción, apoyo y confianza a nuestros clientes y a nuestra gente, siempre innovando.

\section{Visión}

Más clientes, con más productos, transando más.

\section{Valores}

- Honestidad

- Lealtad

- Respeto

- Responsabilidad

- Actitud de servicio

\section{Modelo de competencias de la empresa}

El modelo de gestión por competencias de la entidad que se analizó lleva varios años vigente y se ha trabajado por los subsistemas del área de Talento Humano con éxito. El área busca por medio de él, asegurar que la compañía cuente con el personal adecuado para su buen funcionamiento, teniendo en cuenta que éste se compone de cuatro competencias generales u organizacionales para todos los miembros de la organización y las específicas que se asignan en 
los perfiles del cargo dependiendo del área y funciones que vaya a desempeñar. El área de formación nos permite hacer revisión de las competencias organizacionales con los niveles y comportamientos esperados para cada rol, estos son los siguientes:

- Actitud de cambio e innovación: Capacidad de responder adecuadamente y aceptar lo cambios en la empresa, además, de hallar formas diferentes de hacer las cosas.

- Nivel 1: Está abierto a nuevas ideas y al cambio

- Nivel 2: Se adapta efectivamente al cambio (Apoyo)

○ Nivel 3: Contribuye activamente con el cambio y propone ideas (Contribuyente individual)

○ Nivel 4: Facilita el cambio y la generación de ideas (Líder táctico)

○ Nivel 5: Define una clara visión de cambio e innovación para la organización (Líder estratégico)

- Compromiso con el cliente: Adecuación de una buena vida personal y profesional que creen en su personalidad comportamientos positivos que impacten en el cliente interno y externo positivamente de manera que sea leal con la organización.

○ Nivel 1: Tiene disposición ante el cliente

- Nivel 2: Asegura los procesos para la satisfacción del cliente (Apoyo)

○ Nivel 3: Asesora y da valor agregado al cliente (Contribuyente individual)

○ Nivel 4: Gestiona los procesos de su área con visión de cliente (Líder táctico, líder estratégico)

- Identificación con el banco: Generar sentido de pertenencia y orgullo por el banco, actuando personal y profesionalmente conforme a sus políticas, valores y necesidades.

○ Nivel 1: Respeta la organización

- Nivel 2: Muestra lealtad hacia el banco

○ Nivel 3: Actúa con compromiso hacia el banco (Apoyo, contribuyente individual)

- Nivel 4: Vive y promueve el compromiso con el banco (Líder táctico, líder estratégico)

- Orientación a resultados: Interés por obtener los resultados propuestos, haciendo las cosas bien desde el principio garantizando calidad en cada tarea.

○ Nivel 1: Cumple con los resultados esperados

- Nivel 2: Actúa para superar los resultados (Apoyo) 
○ Nivel 3: Se fija metas retadoras y actúa para lograrlas (Contribuyente individual)

○ Nivel 4: Actúa con visión de costo-beneficio (Líder táctico, líder estratégico)

○ Nivel 5: Emprende acciones adicionales y asumen riesgos calculados para asegurar el logro de los resultados del banco

\section{Programa de inducción general actual}

La directora del área de formación de la empresa facilitó la revisión del proceso de inducción general, el cual consta de dos días: El primero presencial, donde un formador da la información necesaria a los colaboradores y en el segundo, virtual, donde los colaboradores ingresan a la intranet para realizar los cursos de normatividad requeridos.

El contenido brindado por los formadores en el primer día de inducción es el siguiente:

1. Bienvenida y proyección de un video del presidente de la empresa, quien da unas palabras de bienvenida a los nuevos trabajadores.

2. Por medio de una socialización se pide a los colaboradores que se presenten y den a conocer los siguientes aspectos:

○ Nombre

- Personaje o caricatura con que se identifica y porqué

○ Cómo llegó al banco

○ Hobbies

- A dónde quieren llegar dentro de la organización.

3. Metodología de los dos días de la inducción.

4. En una socialización se explica cómo está conformado el sistema financiero en el país (Jerarquía y organismos que lo componen).

5. Por medio de vídeos se dan a conocer las empresas socias, y la solidez del grupo que conforman.

6. Características de la empresa que dan convicción al trabajador: Experiencia, trayectoria, adaptabilidad, respaldo económico, seriedad, unidad de acción y división.

7. Historia de la organización (Trayectoria desde su fundación, hasta su situación actual) 
8. Estructura organizacional, áreas y sus funciones.

9. Cobertura de la empresa en cuanto a ubicación geográfica y canales.

10. Se introduce a la cultura organizacional del banco dando a conocer lo que se espera de los colaboradores, en lo que se les pide acoplar los modos de ser, hacer y actuar de la organización.

11. Misión, visión y valores.

12. Iniciativas para la incorporación de la cultura organizacional:

○ Modelo de Talento Humano

○ Cultura del servicio

- Comunicaciones

13. Medios de comunicación interna de la organización.

14. Introducción al modelo de gestión por competencias, de la siguiente forma:

○ Vídeo sobre valores generales.

- Definición y conceptos sobre competencias y gestión por competencias.

- Competencias organizacionales

15. Evaluación de desempeño de la empresa, explicando los siguientes aspectos:

- Conceptos sobre evaluación de desempeño 360.

- Aplicación y metodología de la evaluación de desempeño 360 en la empresa

16. Importancia de la excelencia del servicio para la empresa, se explican los siguientes aspectos:

○ Introducción al servicio al cliente para la empresa.

- Frentes estratégicos del servicio

- Cultura interna del servicio

- Calidad en el servicio

- Experiencia del cliente

- Programa de observación de los aspectos a medir y evaluar la calidad del servicio: Errores en los procesos, actitud y mejora continua.

- Buenas costumbres de cortesía y atención.

- Métodos de evaluación del servicio al colaborador y su relación con la evaluación del desempeño.

- Modelos y herramientas para asegurar la calidad del servicio. 
- Ciclo de atención al cliente.

- Tips para una buena atención al cliente vía telefónica.

17. Segmento de clientes y portafolio de productos y servicios.

En el segundo día de inducción general de los nuevos colaboradores, se realizan los cursos que se exige a nivel normativo e interno que todos los colaboradores conozcan. Tras realizar la lectura de la información debe aprobar algunas evaluaciones que aseguran que la interiorice. Los temas de los cursos son:

- Evaluación de la información recibida en la inducción

- SAC (Sistema de atención al cliente financiero)

- SARLAFT (Sistema de Administración de Riesgo del Lavado de Activos)

- Código de ética de la empresa

- Código del Buen Gobierno

- SARO (Sistema de Administración de Riesgo Operativo)

- Seguros Fogafín

- Manejo de la intranet de la empresa

- Plan de continuidad del negocio

- Ley SOX

\section{Resultados}

Con las entrevistas semiestructuradas (Ver anexo 1) se entendió que el área de formación desea diseñar un programa de inducción general al banco que incremente la motivación y sentido de pertenencia por la empresa desde el primer día, mejorando varios de sus aspectos, tales como:

- Generar en su manejo estrategias que motiven las personas desde el primer día destacando aspectos del banco como que es una empresa seria, rentable, que da el respaldo económico necesario a sus miembros, pertenece a uno de los grupos financieros 
más grandes del país, además de estar internacionalizándose, es una empresa joven y tecnológica que se ajusta rápido al cambio.

- Disminuir el contenido y la información presentada, teniendo en cuenta que es necesario que se incluya la siguiente: La historia, la planeación estratégica del banco, los beneficios, forma adecuada de contestar el teléfono, métodos de evaluación del servicio, a la empresa la estructura organizacional, el modelo de gestión por competencias, los vídeos de la historia del banco y de bienvenida del presidente.

- Crear un protocolo de bienvenida en el área

No se realizaba evaluación de la información recibida en la inducción general para los colaboradores, por lo que se plantea la posibilidad de implementarla.

Además de esto, el programa de inducción pretende que los colaboradores conozcan y apropien desde el primer día las competencias organizacionales, sin embargo, aspectos como las competencias específicas del cargo, la evaluación de competencias y de servicio son muy teóricos y tendrían un mejor impacto si fuera su jefe inmediato quien los instruyera.

La empresa realizó un "Focus group" con 20 personas que recibieron la inducción en el transcurso de los dos últimos dos años y llegaron a la conclusión de que les hubiera gustado contar en el momento de su llegada a la empresa con:

$\checkmark$ Una bienvenida estelar

$\checkmark$ Conocer los beneficios del banco de una forma mejor

$\checkmark$ Una bienvenida en el área

$\checkmark$ Que se les asigne un compañero que los acompañe y guíe en sus procesos durante el proceso de acoplamiento

$\checkmark$ Tener acceso a las aplicaciones y elementos de área

De una encuesta tipo Likert aplicada a colaboradores que ingresaron a la organización en el transcurso del año (Ver anexos 2 y 3 ) se obtuvieron las siguientes respuestas: 
Tabla 2: Resultados de la encuesta a colaboradores

\begin{tabular}{|c|c|c|c|c|c|c|c|}
\hline Respuesta & $\mathbf{1}$ & $\mathbf{2}$ & $\mathbf{3}$ & $\mathbf{4}$ & $\mathbf{5}$ & $\mathbf{6}$ & $\mathbf{7}$ \\
\hline Totalmente de acuerdo & $38,9 \%$ & $27,8 \%$ & $33,3 \%$ & $44,4 \%$ & $16,7 \%$ & $66,7 \%$ & $38,9 \%$ \\
\hline De acuerdo & $55,6 \%$ & $55,6 \%$ & $55,6 \%$ & $44,4 \%$ & $55,6 \%$ & $27,8 \%$ & $50 \%$ \\
\hline En desacuerdo & $5,6 \%$ & $16,7 \%$ & $11,1 \%$ & $11,1 \%$ & $27,8 \%$ & $5,6 \%$ & $11,1 \%$ \\
\hline Totalmente en desacuerdo & $0 \%$ & $0 \%$ & $0 \%$ & $0 \%$ & $0 \%$ & $0 \%$ & $0 \%$ \\
\hline
\end{tabular}

Una gran mayoría de los colaboradores coincidieron en que la información entregada en la inducción es clara y útil, sin embargo, uno de los objetivos de esta es dar a conocer la cultura organizacional, y una de las preguntas con la que están más en desacuerdo las personas encuestadas es la que corresponde a indagar si aspecto fue claro.

La pregunta con la que más se encuentran en desacuerdo los colaboradores es la que indaga si sus expectativas en la organización se cumplieron. Los ítems evaluados en las demás preguntas correspondientes al entendimiento de las competencias de la empresa, la motivación por trabajar al finalizar la inducción general, la alineación y bienvenida al ingresar al área tienen un bajo grado de insatisfacción.

\section{Discusión de resultados}

El programa de inducción general de la empresa se enfoca en dar la información necesaria a los nuevos colaboradores, pero no se había considerado el enfoque a la motivación por medio de ella, tampoco se había evaluado el impacto que les generaba. Así que en la propuesta se reduce el contenido, para orientarla en entregar la información que es indispensable, usando algunas estrategias que permitan interiorizarla y conectar las necesidades personales de los colaboradores con la organización., teniendo en cuenta que cada ítem se relaciona con las competencias de la empresa. También, aquella información que no es obligatoria durante la inducción general es entregada por medio de un manual de inducción en la herramienta virtual. 
La empresa no cuenta con una estructura para el programa de inducción específica al cargo definido, (Excepto para aquellos que tienen cargos en la red de oficinas) por lo que se crea un protocolo de bienvenida al área que asegure un impacto positivo en el colaborador y mantenga la expectativa que se genera en la inducción general. Además de esto allí se le asigna un tutor, que será alguien de su área y se encargará darle un acompañamiento durante un mes, en el que le explicará las funciones que desempeñará y sus herramientas de trabajo.

Se crea un método de evaluación para el área que permita conocer la perspectiva de los colaboradores de la inducción, y otro la llegada al área, el cual se aplica después de un mes de haber ingresado allí.

\section{Propuesta para el nuevo programa de inducción general}

\section{Contenido de la inducción general}

El primer paso dentro de la organización para un colaborador es la inducción general sobre la empresa, este paso se compone de tres días: Dos presenciales y uno virtual. Los contenidos son los siguientes:

Tabla 3: Propuesta, metodología de la inducción general

\begin{tabular}{|c|c|c|c|}
\hline Actividad & Metodología sugerida & Objetivo & $\begin{array}{c}\text { Competencia } \\
\text { inducida }\end{array}$ \\
\hline \multicolumn{4}{|c|}{ Día 1 } \\
Bienvenida a \\
\begin{tabular}{c|c|c|c|} 
los nuevos \\
colaboradores
\end{tabular} & $\begin{array}{c}\text { Se tiene para cada colaborador } \\
\text { preparados en su silla una carta de } \\
\text { bienvenida con su nombre y un } \\
\text { desayuno o aperitivo, además de un } \\
\text { cartel en el salón con un aviso de } \\
\text { bienvenida. Las directoras de } \\
\text { Talento Humano se turnarán las }\end{array}$ & $\begin{array}{c}\text { Hacer que el } \\
\text { colaborador } \\
\text { sienta que la } \\
\text { empresa } \\
\text { esperaba su } \\
\text { llegada y se }\end{array}$ & $\begin{array}{c}\text { Identificación con el } \\
\text { banco }\end{array}$ \\
\hline
\end{tabular}




\begin{tabular}{|c|c|c|c|}
\hline & $\begin{array}{l}\text { inducciones para ir a dar un } \\
\text { mensaje de acogida. }\end{array}$ & $\begin{array}{l}\text { agrada por la } \\
\text { misma. }\end{array}$ & \\
\hline $\begin{array}{c}\text { Vídeo de } \\
\text { bienvenida del } \\
\text { presidente }\end{array}$ & $\begin{array}{l}\text { Se presenta el vídeo de bienvenida } \\
\text { a la empresa por el presidente y se } \\
\text { hace una socialización para conocer } \\
\text { el impacto de la intervención del } \\
\text { directivo para su llegada }\end{array}$ & $\begin{array}{l}\text { Transmitir que } \\
\text { el máximo } \\
\text { directivo se } \\
\text { interesa por su } \\
\text { llegada. }\end{array}$ & $\begin{array}{l}\text { Identificación con el } \\
\text { banco }\end{array}$ \\
\hline $\begin{array}{l}\text { Actividad de } \\
\text { activación }\end{array}$ & $\begin{array}{l}\text { Se tienen listas unas tarjetas con } \\
\text { algún dibujo cortadas a la mitad (La } \\
\text { cantidad de tarjetas completas debe } \\
\text { ser la mitad de los asistentes). Cada } \\
\text { persona saca una mitad y debe } \\
\text { reunirse con la persona que tenga la } \\
\text { otra mitad de su dibujo. Entre ellos } \\
\text { socializan su nombre, hobbies, } \\
\text { sueños y cómo creen que el banco } \\
\text { puede ayudar a lograrlos. Al } \\
\text { finalizar cada colaborador se pone } \\
\text { de pie y expone las características } \\
\text { de sus compañeros. }\end{array}$ & $\begin{array}{c}\text { Activar al } \\
\text { grupo, conocer } \\
\text { a los nuevos } \\
\text { colaboradores y } \\
\text { que se conozcan } \\
\text { entre ellos }\end{array}$ & $\begin{array}{c}\text { Identificación con el } \\
\text { banco }\end{array}$ \\
\hline $\begin{array}{l}\text { Dar a conocer } \\
\text { las } \\
\text { características } \\
\text { de la empresa }\end{array}$ & $\begin{array}{c}\text { El formador da a conocer las } \\
\text { cualidades de la empresa en cuanto } \\
\text { a su rentabilidad, logros, trayectoria } \\
\text { y adaptabilidad. Además, de los } \\
\text { aspectos que generan confiabilidad } \\
\text { en el trabajador; socializando con } \\
\text { los asistentes el impacto que esto } \\
\text { les genera. }\end{array}$ & $\begin{array}{l}\text { Generar } \\
\text { confiabilidad } \\
\text { por la empresa y } \\
\text { aterrizar las } \\
\text { expectativas que } \\
\text { tienen en ella }\end{array}$ & $\begin{array}{c}\text { Identificación con el } \\
\text { banco/Actitud de } \\
\text { cambio e } \\
\text { innovación }\end{array}$ \\
\hline $\begin{array}{l}\text { Vídeo del } \\
\text { grupo } \\
\text { financiero }\end{array}$ & $\begin{array}{l}\text { Se proyecta el vídeo del grupo } \\
\text { financiero al que pertenece la } \\
\text { empresa, siendo este uno de los más } \\
\text { grandes del país, enseñando sus } \\
\text { logros y confiabilidad }\end{array}$ & $\begin{array}{c}\text { Motivar al } \\
\text { colaborador al } \\
\text { mostrar que } \\
\text { llegan a un gran } \\
\text { grupo financiero }\end{array}$ & $\begin{array}{l}\text { Identificación con el } \\
\text { banco }\end{array}$ \\
\hline $\begin{array}{c}\text { Presentación } \\
\text { de la } \\
\text { planeación } \\
\text { estratégica }\end{array}$ & $\begin{array}{l}\text { Instruir al grupo sobre la planeación } \\
\text { estratégica (Misión, visión, valores, } \\
\text { objetivos), enfatizando en el "para } \\
\text { qué" de que cada uno de ellos esté } \\
\text { en la empresa. }\end{array}$ & $\begin{array}{l}\text { Demostrar a } \\
\text { cada } \\
\text { colaborador que } \\
\text { sus funciones } \\
\text { tienen un }\end{array}$ & $\begin{array}{l}\text { Orientación a } \\
\text { resultados }\end{array}$ \\
\hline
\end{tabular}




\begin{tabular}{|c|c|c|c|}
\hline & & $\begin{array}{c}\text { objetivo en la } \\
\text { empresa }\end{array}$ & \\
\hline $\begin{array}{c}\text { Vídeo de la } \\
\text { historia del } \\
\text { banco }\end{array}$ & $\begin{array}{l}\text { Se proyecta el vídeo de la historia } \\
\text { del banco, seguido de socializar sus } \\
\text { logros, avances y el segmento de } \\
\text { mercado y productos que se ofrecen } \\
\text { en la actualidad, además de } \\
\text { proyectos futuros. }\end{array}$ & $\begin{array}{c}\text { Enseñar al } \\
\text { grupo la } \\
\text { trayectoria de la } \\
\text { empresa, } \\
\text { posición y } \\
\text { proyectos } \\
\text { actuales }\end{array}$ & $\begin{array}{c}\text { Actitud de cambio e } \\
\text { innovación }\end{array}$ \\
\hline $\begin{array}{c}\text { Estructura } \\
\text { organizacional } \\
\text { de la empresa }\end{array}$ & $\begin{array}{c}\text { Se proyecta el boceto de la } \\
\text { estructura organizacional con un } \\
\text { signo de pregunta en cada cuadro. } \\
\text { El formador empieza a mostrar lo } \\
\text { que llevan, uno por uno. En cada } \\
\text { cuadro se halla el nombre del área y } \\
\text { el nombre del (os) colaborador (es) } \\
\text { que se dirige a esa área. } \\
\text { Cuando aparezca el nombre del } \\
\text { colaborador, este dice lo que sabe } \\
\text { del área y el formador lo completa. } \\
\text { El formador sustenta las áreas a las } \\
\text { que no va ningún colaborador. }\end{array}$ & $\begin{array}{l}\text { Hacer conocer } \\
\text { las áreas de la } \\
\text { empresa, los } \\
\text { objetivos y la } \\
\text { forma en que } \\
\text { interactúan }\end{array}$ & $\begin{array}{l}\text { Orientación a } \\
\text { resultados }\end{array}$ \\
\hline $\begin{array}{c}\text { Explicación } \\
\text { de los } \\
\text { beneficios del } \\
\text { banco }\end{array}$ & $\begin{array}{l}\text { Se proyecta un árbol que en sus } \\
\text { ramas tenga los nombres de los } \\
\text { beneficios del banco y se pide a los } \\
\text { colaboradores que lo observen y } \\
\text { definan aquellos beneficios que más } \\
\text { se ajustan a sus necesidades o más } \\
\text { llaman su atención. } \\
\text { Seguido de esto se les hace entrega } \\
\text { de algunos posts it donde escriben } \\
\text { su nombre y lo pegan en aquellos } \\
\text { beneficios que eligieron. } \\
\text { En una socialización cada persona } \\
\text { indica porqué le llama la atención } \\
\text { ese beneficio; paulatinamente el } \\
\text { formador va explicando en qué }\end{array}$ & $\begin{array}{l}\text { Hacerlos notar } \\
\text { que el banco se } \\
\text { interesa por su } \\
\text { satisfacción } \\
\text { personal }\end{array}$ & $\begin{array}{l}\text { Identificación con el } \\
\text { banco }\end{array}$ \\
\hline
\end{tabular}




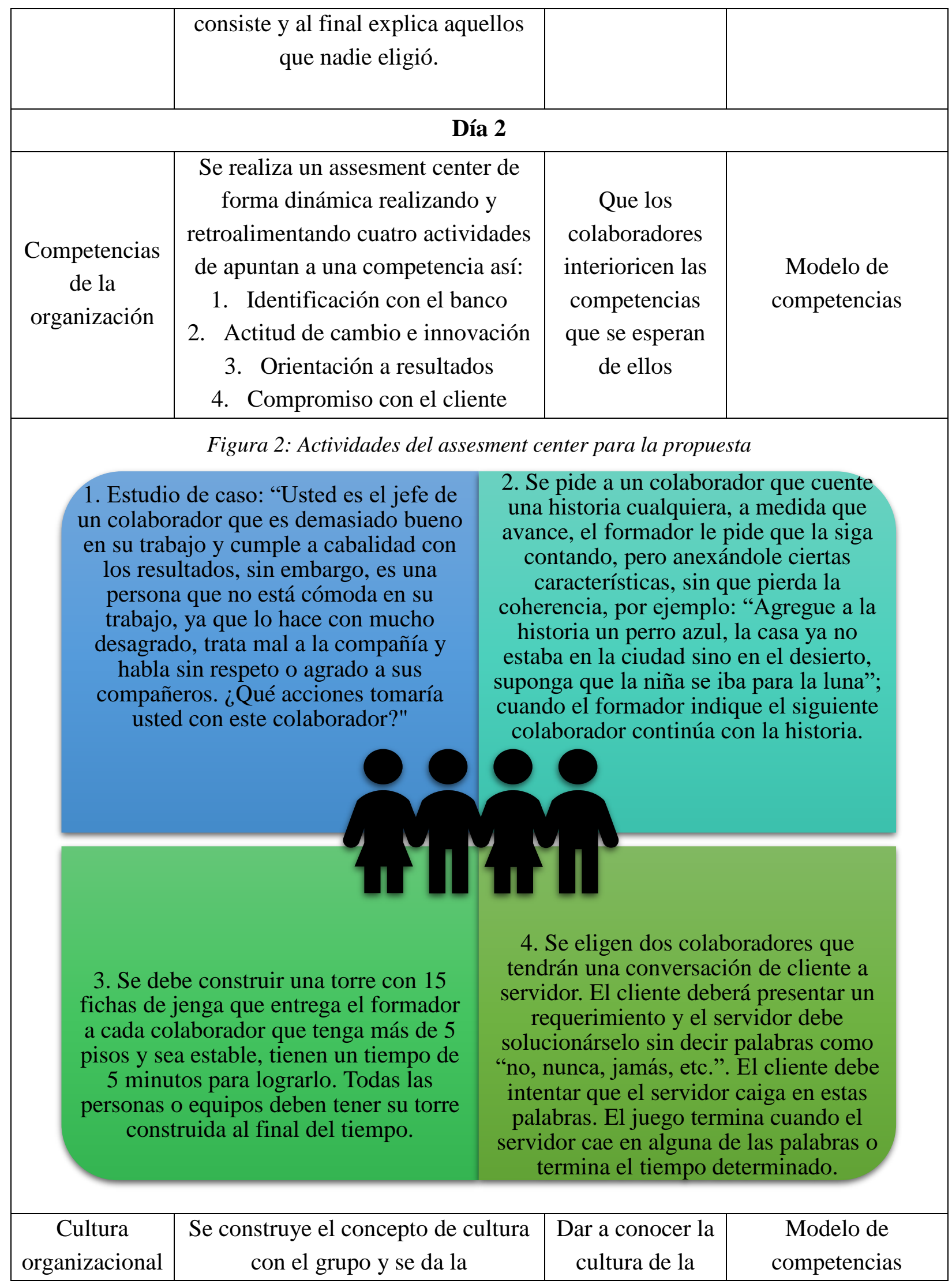




\begin{tabular}{|c|c|c|c|}
\hline & $\begin{array}{l}\text { retroalimentación de la actividad } \\
\text { realizada explicando cómo son los } \\
\text { colaboradores en la organización de } \\
\text { acuerdo con las competencias } \\
\text { evaluadas anteriormente }\end{array}$ & $\begin{array}{l}\text { empresa y las } \\
\text { características } \\
\text { de los } \\
\text { colaboradores }\end{array}$ & \\
\hline $\begin{array}{l}\text { Servicio el } \\
\text { cliente en la } \\
\text { empresa }\end{array}$ & $\begin{array}{l}\text { Se proyectan los métodos de } \\
\text { evaluación del servicio al cliente en } \\
\text { columna, como se muestra en el } \\
\text { gráfico, luego se entregan a los } \\
\text { participantes, por grupo o persona } \\
\text { (según la cantidad), un afiche con } \\
\text { cada definición. } \\
\text { Los participantes deben ubicar las } \\
\text { definiciones en frente del método } \\
\text { que crean conveniente. } \\
\text { Finalmente, el formador corrige la } \\
\text { actividad y la retroalimenta } \\
\text { resaltando la importancia del } \\
\text { servicio al cliente para el banco. }\end{array}$ & $\begin{array}{l}\text { Enseñar la } \\
\text { importancia que } \\
\text { tiene el cliente } \\
\text { en la empresa }\end{array}$ & $\begin{array}{l}\text { Compromiso con el } \\
\text { cliente }\end{array}$ \\
\hline $\begin{array}{l}\text { Pautas para } \\
\text { contestar el } \\
\text { teléfono }\end{array}$ & $\begin{array}{c}\text { Se dan los pasos para contestar el } \\
\text { teléfono y el formador a cargo } \\
\text { simula una llamada sencilla con } \\
\text { cada colaborador para asegurar que } \\
\text { hayan acatado las pautas }\end{array}$ & $\begin{array}{c}\text { Asegurar un } \\
\text { servicio } \\
\text { telefónico } \\
\text { adecuado desde } \\
\text { el inicio } \\
\end{array}$ & $\begin{array}{l}\text { Compromiso con el } \\
\text { cliente }\end{array}$ \\
\hline Finalización & $\begin{array}{c}\text { Se finaliza preguntando a cada } \\
\text { colaborador lo que le quedó de la } \\
\text { inducción y la perspectiva generada } \\
\text { sobre la empresa }\end{array}$ & $\begin{array}{l}\text { Cerrar el } \\
\text { proceso }\end{array}$ & \\
\hline \multicolumn{4}{|c|}{ Día 3} \\
\hline $\begin{array}{l}\text { Cursos de } \\
\text { normatividad } \\
\text { y encuesta de } \\
\text { satisfacción } \\
\quad \text { de la } \\
\text { inducción }\end{array}$ & $\begin{array}{l}\text { Creación del usuario para la } \\
\text { plataforma de formación virtual y } \\
\text { explicar la realización de cursos de } \\
\text { normatividad, iniciando por } \\
\text { diligenciar una encuesta de } \\
\text { satisfacción sobre la inducción } \\
\text { recibida }\end{array}$ & $\begin{array}{l}\text { Cerrar la } \\
\text { inducción } \\
\text { general y } \\
\text { conocer el } \\
\text { impacto de la } \\
\text { inducción. }\end{array}$ & \\
\hline
\end{tabular}




\section{Información entregada en el manual de inducción virtual}

Tabla 4: Propuesta, manual de inducción virtual

\begin{tabular}{|c|c|}
\hline \multicolumn{2}{|r|}{ Inducción. Parte virtual } \\
\hline \multirow{6}{*}{ Temas } & Explicación de la estructura del sistema financiero en Colombia \\
\hline & Ubicación geográfica de la empresa a lo largo del territorio \\
\hline & Canales de atención al cliente \\
\hline & Medios de comunicación interna de la organización \\
\hline & Presentación de la intranet \\
\hline & $\begin{array}{c}\text { Estrategias de gestión del Talento Humano de la empresa; horarios y } \\
\text { prestaciones sociales de la empresa. }\end{array}$ \\
\hline Actividad & $\begin{array}{l}\text { Tras terminar cada tema se presenta un cuestionario con } 3 \text { preguntas de } \\
\text { selección múltiple. El colaborador no avanzará hasta no responder } \\
\text { adecuadamente. Sin embargo, esto se presenta a modo de refuerzo, no de } \\
\text { evaluación; ya que la evaluación se realiza al final del módulo y comprende los } \\
\text { temas de la inducción presencial y la virtual. }\end{array}$ \\
\hline
\end{tabular}

\section{Protocolo de ingreso al área}

Cuando el colaborador ingresa a su puesto de trabajo el jefe en ayuda del equipo se encargan de llevar a cabo un protocolo para dar la bienvenida e inducción en el área al nuevo integrante, el protocolo es el siguiente:

- Queda al libre albedrío del equipo la decoración del puesto de trabajo o el dar algún alimento para su bienvenida, sin embargo, se pide que se anexe un afiche de felicitaciones que envía el área de Talento Humano. Además, de realizar una actividad de socialización que consiste en que cada miembro dejará en un recipiente sobre el puesto en un papel su nombre y una palabra que lo identifique.

- El equipo completo da la bienvenida

- El jefe inmediato da al colaborador un recorrido por el área para conocer a sus compañeros, lo que cada uno realiza en el área y la forma en que interactuarán. Cuando 
se encuentre con cada compañero, este último le da una explicación de la palabra escrita en el papel, esto con el fin de que interactúen a un nivel más personal.

- El primer miembro del equipo con que tiene esta interacción será con su jefe, allí además de lo ya mencionado, se explican algunos temas que anteriormente se daban en la inducción, pero, se estima que tendrían más impacto si los explica su jefe:

- Da el espacio para realizar el módulo del manual de inducción de la empresa

○ Le explica sus funciones y la relevancia de su cargo en el área

- Se enfatiza en las competencias específicas que requiere su cargo, explicando que se evalúa periódicamente su desempeño por competencias y la metodología de dicho proceso

- Se profundiza en la importancia del servicio al cliente y los métodos de evaluación de este

- Explica el manejo del plan carrera de la organización

○ Le presenta al compañero que estará a cargo durante el próximo mes de enseñarle el uso de las herramientas, aplicaciones y funciones.

- Un mes después el colaborador realizará en la herramienta virtual una encuesta de satisfacción sobre la inducción a su puesto de trabajo

\section{Encuestas de satisfacción}

La primera encuesta de satisfacción se realiza al finalizar el día de la jornada de inducción general con el objetivo de conocer el impacto que ha logrado en los colaboradores y la claridad de la información, se realiza con opciones de respuesta en una escala de 1 a 5, donde 1 es la más baja y 5 la más alta. Consta de las siguientes preguntas:

Tabla 5: Encuesta de satisfacción a la inducción general

\begin{tabular}{|c|c|c|c|c|c|}
\hline Preguntas & $\mathbf{1}$ & $\mathbf{2}$ & $\mathbf{3}$ & $\mathbf{4}$ & $\mathbf{5}$ \\
\hline ¿Considera clara la información entregada en la inducción & & & & & \\
general? & & & & & \\
\hline
\end{tabular}




\begin{tabular}{|c|l|l|l|l|l|}
\hline ¿Fueron adecuadas las condiciones del lugar? & & & & & \\
\hline ¿Fue apropiada la duración del curso? & & & & & \\
\hline ¿Fue correcto el manejo del curso por la persona a cargo? & & & & & \\
\hline ¿Es clara la cultura organizacional del banco? & & & & & \\
\hline ¿Se alinea lo presentado sobre la empresa con sus & & & & & \\
necesidades personales? & & & & \\
\hline Comentarios o sugerencias sobre el proceso recibido: & \\
\hline
\end{tabular}

La segunda encuesta se aplica un mes después de que el colaborador ingresa al área para conocer su impresión sobre la empresa durante los primeros días de trabajo y asegurar que se esté dando una adecuada inducción específica al cargo. Sus respuestas constan de una calificación en una escala de 1 a 5 , donde 1 es la calificación más baja y 5 la más alta. Las preguntas son las siguientes:

Tabla 6: Encuesta de satisfacción a la inducción específica

\begin{tabular}{|c|c|c|c|c|c|}
\hline Pregunta & $\mathbf{1}$ & 2 & 3 & 4 & 5 \\
\hline ¿Se sintió bien recibido al llegar a su área? & & & & & \\
\hline $\begin{array}{l}\text { ¿Considera que se la ha dado una buena inducción a sus } \\
\text { funciones? }\end{array}$ & & & & & \\
\hline $\begin{array}{c}\text { ¿Se han entregado y explicado las herramientas necesarias } \\
\text { para el cargo? }\end{array}$ & & & & & \\
\hline ¿Ha sido fácil el acoplamiento a su puesto de trabajo? & & & & & \\
\hline ¿Se han cumplido sus expectativas en la empresa? & & & & & \\
\hline ¿Cómo ha sido su relación con sus compañeros de trabajo? & & & & & \\
\hline ¿Cómo ha sido su relación con su jefe inmediato? & & & & & \\
\hline
\end{tabular}




\section{Conclusiones}

La empresa analizada presenta un programa de inducción general, que si bien, se encontró que entrega a los nuevos colaboradores toda la información necesaria, es un proceso bastante extenso y la teoría allí explicada es bastante, por lo que los participantes no la interiorizan por completo; esto ya que el objetivo del área con la inducción era, solamente, asegurar que los nuevos colaboradores ingresaran a su puesto de trabajo conociendo aspectos de la organización que necesitan saber. Además de esto no se hacía seguimiento al proceso para conocer que el impacto generado en los colaboradores fuera significativo.

La empresa no cuenta con un programa de inducción bien estructurado, por lo tanto, los nuevos colaboradores no reciben la información necesaria sobre sus áreas por parte de sus jefes inmediatos o de sus compañeros, por lo que queda al libre albedrío de estos la bienvenida, sin tener en cuenta, que la inducción al nuevo personal es un proceso que involucra al área de Talento Humano y a la que va dirigido el colaborador (Mondy, 2010).

El modelo de competencias del banco es un esquema que muestra las aptitudes que se esperan de las personas para que los procesos funcionen correctamente. Tiene aquellas que se necesitan para cada área en específico dependiendo de las funciones y el objetivo; así como contiene cuatro que son transversales para todos los miembros de la organización (Actitud de cambio e innovación, compromiso con el cliente, orientación a resultados e identificación con el banco). Ya que la inducción general muchas veces se realiza con personas que van dirigidas a diferentes áreas, allí se trabajan las competencias que aplican para todas las personas de la empresa, dejando las específicas a cargo del jefe del nuevo integrante.

La propuesta para el nuevo programa de inducción general entrega a los nuevos colaboradores la información que se requiere que conozcan. Sin embargo, de manera virtual, se da a conocer aquella información meramente teórica, dando espacio para trabajar de forma presencial los aspectos de la empresa que más impactan y generan motivación del trabajador en el banco, junto con el modelo de competencias y aspectos de la cultura organizacional. Para esto, se implementan varias dinámicas en los diferentes temas, ya que se evidencia que el hecho de manejar los temas de forma lúdica permite que los trabajadores se sientan más motivados para recibir información y hace que esta sea más efectiva, junto con esto, el jugar permite a las 
personas expresar sus ideas y forma de pensar y actuar en medio de un entorno divertido, lo cual genera sentido de pertenencia hacia las organizaciones (Teixes, 2014).

El programa de inducción general pretende presentar a los nuevos colaboradores toda la información que deben conocer al ingresar a su puesto de trabajo, haciendo énfasis en aquellos aspectos que representan un beneficio para darles a entender que han ingresado a una organización sólida y rentable que brinda a sus colaboradores estabilidad económica y seguridad, además de preocuparse de ellos como personas, ya que, cuenta con espacios que generan un ambiente cálido para que los colaboradores sientan que se esperaba su llegada; y de integración que les posibilita socializar entre ellos. Esto, junto con la metodología dinámica genera desde el principio sentido de pertenencia hacia la organización.

Con esto, finalmente, se entregan a las áreas colaboradores motivados e identificados con la empresa que tienen actitud para dar inicio al desempeño de sus labores de una forma óptima, de manera que el impacto en el área y la empresa sea el esperado. Para este mismo objetivo, también se dan a conocer e interiorizar las competencias que se esperan de cada persona, trabajándolas en una dinámica que llevan a los nuevos trabajadores a ponerlas en práctica simulando ambientes en que son necesarias para dar respuesta a determinada situación.

La presente investigación da a conocer la importancia y necesidad de uno de los primeros pasos en la gestión humana dentro de una organización, como lo es la inducción de personal; el cual es uno de los comienzos de las cadenas de procesos que mantienen a las empresas en funcionamiento. Los gestores de todos los procesos de una empresa son las personas, siendo ellas quienes comandan las máquinas y las técnicas para mantenerla en funcionamiento (Alles, 2008, p: 33), de forma que una persona correctamente preparada hará su gestión correctamente. Dentro de la administración de las empresas se hace necesario tener a las personas debidamente formadas y motivadas, ya que esto, es el inicio para desempeñar su cargo correctamente y que la cadena de procesos que componen las compañías funcione apropiadamente. Este trabajo es una herramienta que enseña las pautas necesarias para diseñar una inducción general que entregue la información que una empresa desea a los nuevos colaboradores mientras se motivan en el proceso. 


\title{
Recomendaciones
}

Para cumplir los objetivos de la inducción general se hacen las siguientes recomendaciones:

1. Evitar explicaciones demasiado extensas por parte del formador a cargo de la inducción.

2. Realizar la retroalimentación y socialización al finalizar cada actividad para garantizar que se ha entendido el objetivo y el tema trabajado.

3. Dar a conocer a los colaboradores el porqué de las competencias que se esperan de ellos, explicando el impacto de estas en la planeación estratégica de la empresa.

4. El jefe inmediato debe dar conocimiento del impacto e importancia del colaborador y el área en el desempeño de la empresa.

5. Incluir en la evaluación virtual de la inducción general los aspectos que representan beneficios para los colaboradores asegurando su entendimiento.

6. La propuesta es flexible, por lo que se deben ir haciendo los ajustes tras las oportunidades de mejora que se encuentren en su implementación.

\begin{abstract}
Anexos
Anexo 1: Herramientas de recolección de información (Entrevista)

Se aplicó una entrevista semiestructurada a la directora del área y la analista de contenidos con el objetivo de precisar las necesidades que los llevaron a realizar cambios en el programa de inducción y la información necesaria para construir el nuevo. Las preguntas bases para la entrevista son:
\end{abstract}

1. ¿Cuál es el principal objetivo del programa de inducción general para los nuevos colaboradores?

2. ¿De qué forma se miden los resultados de la inducción? 
3. ¿Cuál es el resultado que ha generado el programa de inducción en el desempeño de los nuevos colaboradores?

4. ¿Qué oportunidades de mejora se evidencian en el actual programa de inducción?

5. ¿Qué los lleva a realizar cambios en el programa de inducción?

6. ¿Qué expectativas se tienen sobre el nuevo programa de inducción general?

7. ¿Qué temas debe contener el nuevo programa de inducción?

8. ¿De qué forma se incluye el modelo de competencias en el programa de inducción?

9. ¿Cuál es el perfil general del colaborador que pretende tener la empresa por medio del modelo de competencias?

10. ¿Qué aspectos de la cultura organizacional se hace necesario incluir dentro del programa de inducción?

11. ¿De qué forma se puede crear sentido de pertenencia hacia la empresa desde el primer día en la inducción?

12. ¿Cuáles son las fortalezas del banco que se pueden resaltar durante la inducción para generar mayor identificación del nuevo trabajador con la empresa?

13. ¿Considera útil los siguientes métodos para utilizarlos en el manejo de la inducción para cumplir con los objetivos propuestos:

○ Un manual de inducción

○ Un ritual de bienvenida al área

- Dinámicas para la explicación de los temas

- Dar a conocer el plan carrera de la empresa

- Conocer y aterrizar las expectativas del colaborador

- Dar a conocer los horarios y prestaciones de la empresa

- Métodos de evaluación de bienvenida a los colaboradores

\section{Anexo 2: Herramientas de recolección de información (Encuesta)}

Se aplicó una encuesta tipo Likert a colaboradores de la organización que recibieron inducción en el año 2017, con el objetivo de conocer la percepción que tuvieron de la inducción general y la claridad de la información recibida. Las preguntas son las siguientes: 
1. Considero clara y concisa la información presentada en el proceso de inducción

2. Comprendí la cultura organizacional de la empresa durante el proceso de inducción

3. Reconocí las competencias que se esperaban de mí en la empresa con el proceso de inducción

4. Se cumplieron mis expectativas en la empresa al finalizar la inducción

5. Me sentí motivado por trabajar en la empresa al finalizar el proceso de inducción

6. Fui recibido adecuadamente al ingresar a mi puesto de trabajo

7. Estuve alineado con la empresa al iniciar con mis labores en la empresa

Las opciones de respuesta para cada pregunta son las siguientes:

- Totalmente de acuerdo

- De acuerdo

- En desacuerdo

- Totalmente en desacuerdo

\section{Anexo 3: Evidencias de las encuestas aplicadas}

Se adjunta muestra del gráfico que señala un resumen de las personas que realizaron la encuesta.

Figura 3: Anexo: Participantes de la encuesta

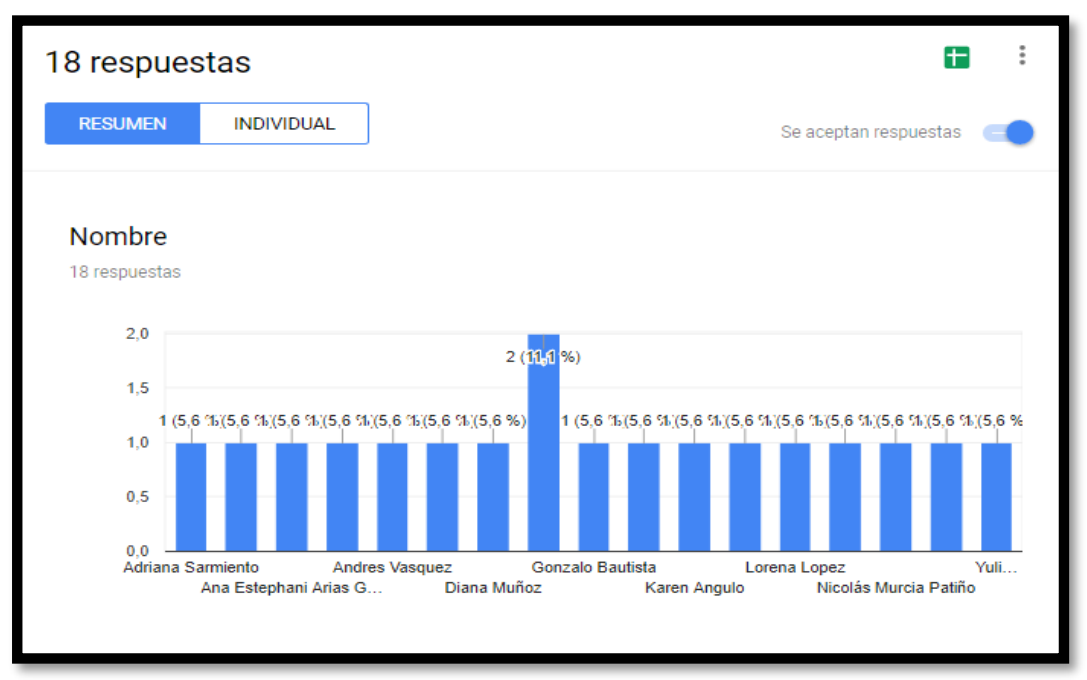


Se adjuntan los gráficos de las preguntas a las que más presentaron inconformidad los colaboradores encuestados:

Figura 4: Pregunta 2 "Comprendí la cultura organizacional de la empresa con el proceso de inducción"

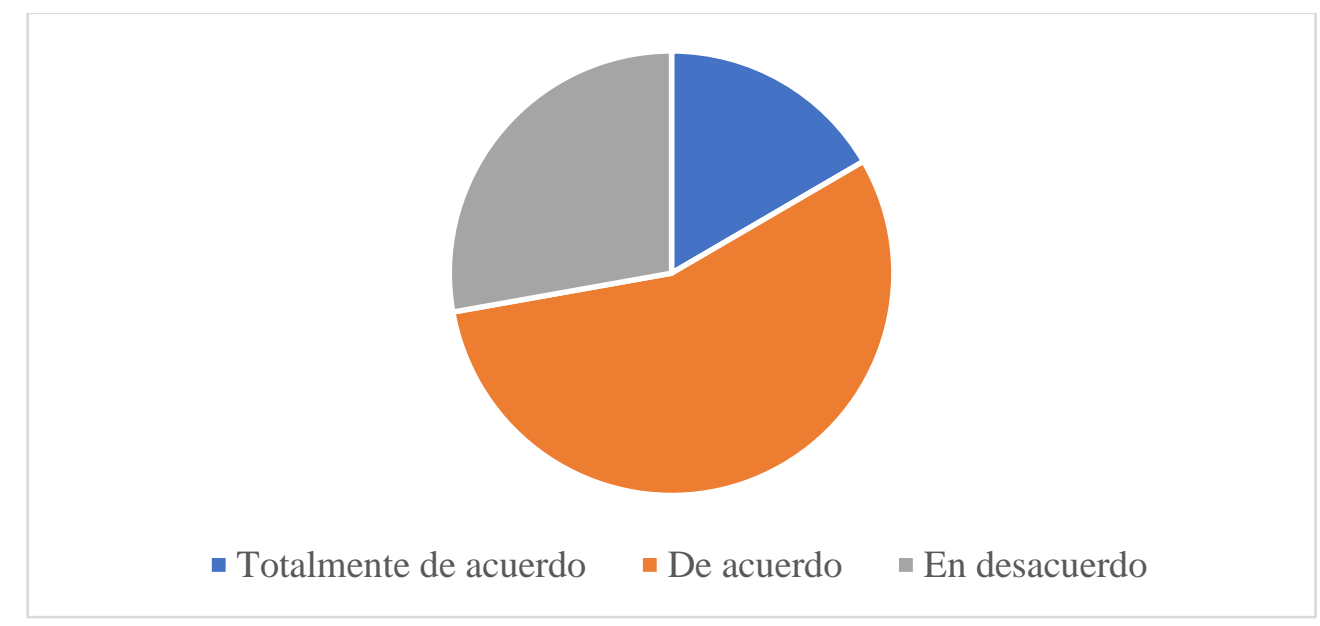

Figura 5: Pregunta 4: "Se cumplieron mis expectativas en la empresa generadas en la inducción

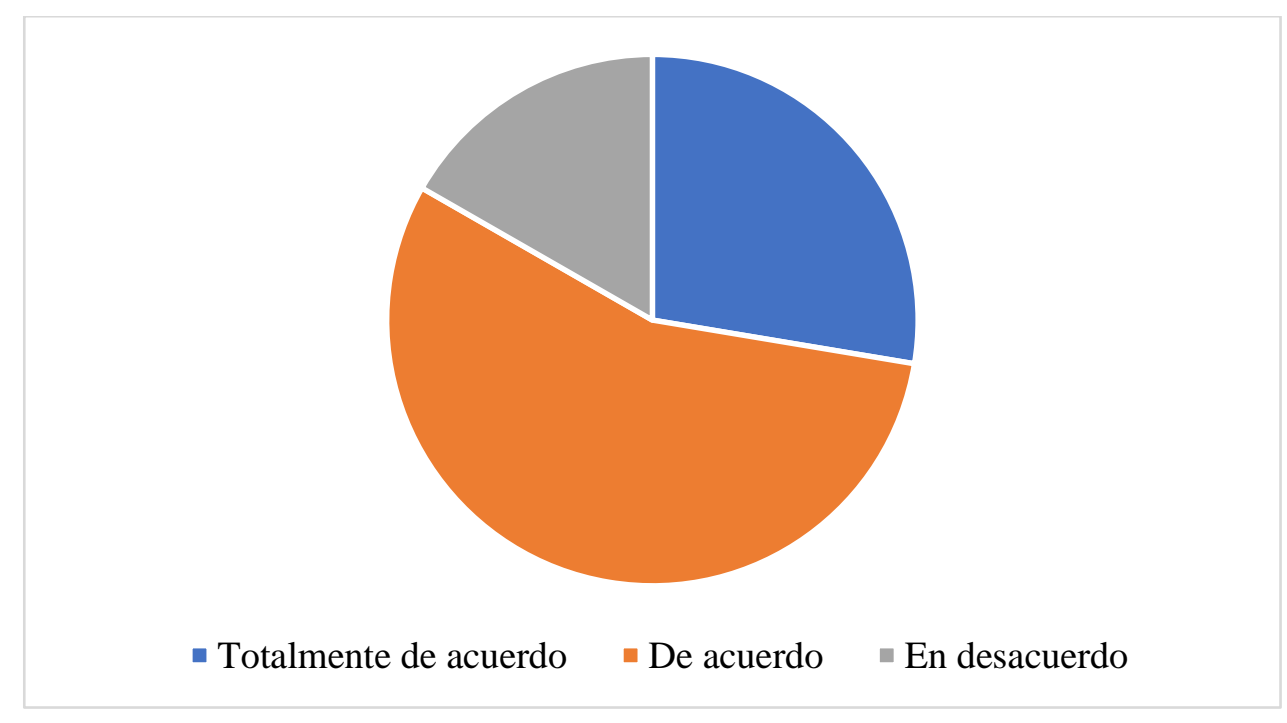




\section{Referencias Bibliográficas}

- Alles M. (2006). Dirección estratégica de recursos humanos: Gestión por competencias (2da edición). Buenos Aires, Argentina: Ediciones Granica.

- Alles M. (2008) Comportamiento organizacional: Cómo lograr un cambio cultural a través de gestión por competencias (4 ${ }^{a}$ edición). Buenos Aires, Argentina: Ediciones Granica

- Alles M. (2010). Conciliar vida profesional y personal: Dos miradas: Organizacional e individual. Buenos Aires, Argentina: Ediciones Granica

- Alles M (2016). Cuestiones sobre gestión de personas: Qué hacer para resolverlas. Buenos Aires, Argentina: Ediciones Granica.

- Alles M. (2016). Dirección estratégica de recursos humanos: Gestión por competencias. Vol. 1 3ra edición. Buenos Aires, Argentina: Ediciones Granica.

- Calvo P. (2014). Gestión por competencias como factor de mejora de calidad asistencial. Madrid, España: Ediciones Díaz de Santos.

- Chávez G. (2002). Manual para el diseño de normas de competencia laboral. México: Panorama editorial.

- Chiavenato I. (2009). Gestión del Talento Humano. México: McGraw-Hill

- Cuesta A. (2010). Gestión del talento humano y del conocimiento. Bogotá, Colombia: Ecoe Ediciones.

- Dessler G. (2001). Administración de personal. Recuperado de https://books.google.com.co/books?id=00dKezzNEAC\&pg=PA249\&dq=gary+dessler+induccion \&hl=es\&sa=X\&ved=0ahUKEwiogaqbsqv WAhWFQCYKHWoUBTUQ6AEIJDAA\#v=onepage $\& \mathrm{q}=$ gary $\% 20$ dessler $\% 20$ induccion $\underline{\& \mathrm{f}=\mathrm{false}}$

- Grados J. (2012) Reclutamiento: Selección, contratación e inducción del personal (4a edición). Bogotá, Colombia: Ecoe Ediciones

- Hernández R., Fernández C., Baptista C. (2010). Metodología de la investigación (5ta edición). México: McGraw-Hill 
- Juan S., Roussos A. (2010). El focus group como técnica de investigación cualitativa. Documento de trabajo No 254. Recuperado de http://www.ceppia.com.co/Herramientas/Herramientas/Manual-Focus-Group.pdf

- Mondy R. (2010). Administración de recursos humanos. Decimoprimera edición. México: Pearson Educación.

- Ríos Y., Sarnataro S., Soto C. (2003). Perfil sobre el éxito de ejecutivos comerciales en la venta de intangibles bancarios. Aplicación del modelo de competencias. Santiago de Chile, Chile: Red PHARO.

- Robbins S., Judge T. (2006). Comportamiento organizacional (13a. edición). Mexico: Pearson Educación.

- Teixes F. (2014). Gamificación: Fundamentos y aplicaciones. Barcelona, España: Editorial UOC.

- Weihrich H., Koontz H. (1998) Administración. Una perspectiva global (11 a edición). Mexico: McGraw Hill.

- Werther W., Davis K. (2014). Administración de recursos humanos. Gestión del capital humano. México: McGraw Hill. 